\title{
Multilaterale Sonderverbindung als semi-spontane Ordnung: Das Beispiel der Baukooperation und des Franchising
}

Franchising und Baukooperation? Auf den ersten Blick scheint man Äpfel mit Birnen vergleichen zu wollen. Aber nur auf den ersten Blick. Beide Vertragsnetzwerke ${ }^{1}$ weisen Strukturen einer semi-spontanen Ordnung auf, die im Recht als multilaterale Sonderverbindung abgebildet werden kann. Es handelt sich um zwei Typen von Netzwerken, auf die die Pflichtenstruktur der Sonderverbindung unterschiedlich reagiert.

Der Fokus der Untersuchung liegt hier auf den Binnenbeziehungen. Es geht also um das rechtliche Verhältnis derjenigen Beteiligten an Baukooperation und Franchising, die untereinander keine Verträge geschlossen haben, die Auftragnehmer bzw. Franchisenehmer. Während es über die Netzwerkeigenschaften des Franchising eine Vielzahl von innovativen Untersuchungen gibt ${ }^{2}$, gilt dies nicht für die Baukooperation. In den 90er Jahren gab es einen Schub von Arbeiten, die sich mit der Verflechtung von Verträgen in der Baukooperation beschäftigt haben, eine Verbindung zu der Netzwerkdiskussion in anderen Bereichen hat aber nicht stattgefunden. In der Baukooperation wurde die Figur des komplexen Langzeitvertrags entwickelt, dessen Eigenschaften jedoch im Deskriptiven verbleiben. ${ }^{3}$ Nur wenige haben zumindest auf die Gesellschaftsähnlichkeit der Baukooperation hingewiesen. Weiter aufgegriffen wurde diese Idee jedoch nicht. ${ }^{4}$ Die Untersuchung von Binnenbeziehungen in Netzwerken wurde in allen Bereichen vernachlässigt. Obwohl vielfach die rechtliche Relevanz von Netzwerken in Abhandlungen bejaht wird, werden zumeist rechtlich relevante Beziehungen zwischen vertraglich unverbundenen Beteiligten an einem Netzwerk mit wenigen Sätzen kategorisch verneint. ${ }^{5}$ Nimmt man die Netzwerkidee jedoch ernst, kommt man nicht umhin, sich mit den Binnenbeziehungen eingehend zu befassen.

1 Bei Vertragsnetzwerken stehen die bilateralen Vertragsvereinbarungen in einem gewissen Beziehungsgeflecht und erzeugen multilaterale Netzwirkungen vgl. Gunther Teubner (2004) Netzwerke als Vertragsverbund, Baden-Baden: Nomos; Knut Lange (1998) Das Recht der Netzwerke, Heidelberg: Verlag Recht \& Wirtschaft, Rn. 10.

2 Mit dem Franchising befassen sich eingehend Teubner (Fn. 1); Annika Schimansky (2003) Der Franchisevertrag nach deutschem und niederländischem Recht, Tübingen: Mohr \& Siebeck; Mathias Rohe (1998) Netzverträge, Tübingen: Mohr \& Siebeck; Rainer Kulms, (2000) Schuldrechtliche Organisationsverträge in der Unternehmenskooperation, BadenBaden: Nomos.

3 Helmut Schlotke (1990) »Vertikale Verknüpfungen von Unternehmern verschiedener Produktionsstufen durch Qualitätssicherungsysteme und Just-In-Time-Lieferungen - komplexe Langzeitverträge besonderer Art?«, in: Fritz Nicklisch (Hg.) Verträge über Computertechnik in Forschung, Verwaltung, Wirtschaft und Technik, Heidelberg: C.F. Müller, 267-277, 273.

4 »Das Thema scheint nichts herzugeben. Doch trügt der Schein «. So schon Peter Gauch (1982) »Ein Bauwerk - mehrere Unternehmer«, 118 ZBJV, 65 ff., 68. Von einem gesellschaftsähnlichen Rechtsverhältnis spricht auch Schlotke (Fn. 3).

5 Rohe (Fn. 2) 430 ff.; Kulms (Fn. 2) 233; Schimansky (Fn. 2) 117 ff. So auch Knut Lange (2001) Virtuelle Unternehmen, Heidelberg: Recht \& Wirtschaft, Rn. 333. 


\section{Strukturmerkmale der Baukooperation}

Das Bild der modernen Baukooperation ist ein durch starke Arbeitsteilung und Spezialisierung geprägtes komplexes Gefüge technisch-wirtschaftlich miteinander verknüpfter Lieferungen und Leistungen, die in der Regel nur innerhalb eines längeren Zeitraums erbracht werden können ${ }^{6}$. Im Gegensatz zum punktuellen, einmaligen Austauschvertrag, der typischerweise nur zwischen zwei Beteiligten besteht, sind an der Planung und Durchführung von Bauverträgen regelmäßig eine Vielzahl von Akteuren beteiligt, wie Architekten, Sonderfachleute, beratende Ingenieure, Statiker und Bauunternehmer. ${ }^{7}$ Der gewünschte wirtschaftliche Erfolg kann durch Abschluss nur eines Vertrages nicht erreicht werden. ${ }^{8}$ Die Verträge weisen einen Rahmencharakter auf, da sie in der Durchführungsphase ständiger Anpassung bedürfen. Komplexe Anlagen sind schlicht im Voraus in allen Einzelheiten nicht planbar.

Der Auftraggeber kann das Bauprojekt als Ganzes ausschreiben und an einen Bieter vergeben, oder er kann es in einzelne sog. »Lose« aufteilen und an verschiedene Bieter vergeben. Die Bieter können Einzelunternehmer, Arbeitsgemeinschaften oder Konsortien sein, die ihrerseits wieder Aufträge an Subunternehmer vergeben. So kann sich das Bild eines gitterförmigen Netzwerks, seltener das eines Sterns ergeben. Teilt der Auftraggeber das Gesamtbauwerk in Lose auf und vergibt die Lose durch Einzelverträge, ist er quasi die einzige Partei, die mit allen Beteiligten in vertraglicher Beziehung steht. Er trägt dann die Verantwortung für die Verknüpfung der einzelnen Teilleistungen zu einem Ganzen (Schnittstellenrisiko), er trägt die Organisations- und Koordinierungslast allein ${ }^{9}$. Die Unternehmer haften nach der Rechtsprechung bei reiner Einzelbeauftragung nebeneinander und unabhängig voneinander allein für ihre Arbeiten. ${ }^{10}$ Dagegen wird der Auftraggeber mit einer Ausschreibung des Gesamtprojekts der schwierigen Koordinierungsaufgabe enthoben, die die Aufteilung in Lose mit sich bringt. ${ }^{11}$ Bei Großprojekten wird in der Regel eine Arbeitsgemeinschaft (ARGE) als Generalunternehmer Vertragspartner des Auftraggebers. ${ }^{12}$ Solange dem Auftraggeber jedoch mehr als ein Vertragspartner gegenübersteht, der die Gewähr für das Risiko für das Gelingen des »schlüsselfertigen« Gesamtprojekts übernimmt (General- bzw. To-

6 Schlotke (Fn. 3) 384.

7 Fritz Nicklisch und Günter Weick (2001) VOB Teil B, 3. Aufl., München: Beck, Einleitung Rn. 4.

8 Karl Larenz und Manfred Wolf (1997) Allgemeiner Teil des Bürgerlichen Rechts, 8. Aufl. München: Beck, § 23 Rn. 127. Bei großen Anlagebauprojekten können das schon mal 700 Schnittstellen sein.

9 Bernhard Hautkappe (1985) Unternehmenseinsatzformen im Industrieanlagenbau, Heidelberg: Verlag Recht \& Wirtschaft, 27.

10 Für die Frage der gesamtschuldnerischen Haftung jetzt Rechtsprechungsänderung, BGH NJW 2003, 2980.

11 Albrecht Barner (1971) Die Arbeitsgemeinschaft in der Bauwirtschaft als besonderer gesellschaftsrechtlicher Typus, München: Schön, 22; Hautkappe (Fn. 9) 31.

12 Wolfgang Koeble und Martin Schwärzel-Peters (1996) )Gesellschaftsvertragliche Ausgestaltung einer Arbeitsgemeinschaft am Beispiel der Bau-Arbeitsgemeinschaft«, 34 DstR, 1734-1740. 
talübernehmer), verbleibt das Schnittstellenrisiko letztlich bei ihm. ${ }^{13}$ Aus der Koordinierungslast ergibt sich zwangsläufig eine starke Stellung des Auftraggebers. Die Baukooperation weist aber keine streng hierarchische Struktur auf, in der Entscheidungsstränge immer von oben nach unten verlaufen. Aus der Komplexität des Projekts und dem Rahmencharakter der Verträge ergibt sich, »dass bei der Vertragsdurchführung fortlaufend Entscheidungen getroffen werden müssen und zwar durch den Auftraggeber, durch den Auftragnehmer oder - meist - durch beide gemeinsam. $\ll^{14}$ Insbesondere wenn Planung und spätere Realität auseinander fallen, kann das Projekt möglicherweise nur abgeschlossen werden, wenn die auftretenden Probleme bereits während der Ausführung gelöst werden, etwa wenn es um notwendige bauliche Änderungen geht. ${ }^{15}$

Die Erstellung eines Bauwerks erfordert es, dass die Beteiligten kooperieren und zielbezogen zusammenwirken müssen. ${ }^{16}$ Alle Projektbeteiligten sind nicht nur faktisch, sondern auch rechtlich in ein Netz intensiver Kooperation und Kommunikation einbezogen. Neben den jeweiligen Vertragspartnern werden eine Vielzahl der sonstigen Projektbeteiligten durch Konkretisierungen, Änderungen und Störungen betroffen, da die Verträge offen für Konkretisierungen und Änderungen sind und infolge des Langzeitcharakters eine erhöhte Störanfälligkeit gegeben ist. Das hat zur Folge, dass auch ihre vertraglichen Beziehungen mit der neuen Situation in Einklang zu bringen sind. ${ }^{17}$ Dadurch entsteht ein persönliches und vertrauensvolles Beziehungsgeflecht, »ein im Zuge der Abwicklung anpassungsbedürftiges und deshalb in seiner inneren Gestaltung weitgehend offenes Beziehungsgeflecht $\ll^{18}$.

Die Konzeption des komplexen Langzeitvertrags gibt eine Beschreibung der Verflechtung der Bauverträge und stellt die Realität dem Leitbild des Werkvertrags, das dem BGB zugrunde liegt, gegenüber. Das Leitbild des Werkvertragsrechts ist die

13 Vgl. auch Hautkappe (Fn. 9) 31, 33, 35. Der Auftraggeber wird jedoch trotz der Vorteile der Gesamtbeauftragung die Aufteilung in Einzelverträge bevorzugen, wenn sein für die Erstellung des Gesamtbauwerks vorhandenes Know-How besser als das zu erwartende der Unternehmer ist. Dies kann v.a. beim Anlagenbau, bei dem es auf das Funktionieren der Anlage ankommt, der Fall sein. Vgl. Hautkappe (Fn. 9) 26.

14 Nicklisch und Weick (Fn. 7) Einleitung Rn. 4.

15 Zur Ermöglichung einer vertragsbegleitenden Entscheidungsfindung enthalten die Verträge eine Vielzahl unterschiedlich ausgeprägter Mechanismen. Diese zielen teils auf die Herbeiführung einer einverständlichen Entscheidung zwischen den Vertragspartnern, teils informieren sie den anderen Vertragspartner über bestimmte Vorgänge, wie etwa auftretende Störungen, und geben ihm damit die Möglichkeit einer entsprechenden Reaktion; teils zielen sie auf klare Feststellungen über Verantwortlichkeiten, Zusatzaufträge usw. Konkrete Beispiele für Mechanismen dieser Art sind Prüfungs- und Hinweispflichten des Auftragnehmers, wenn der Auftraggeber Daten gesetzt, Materialien oder Teile geliefert hat, weiterhin Rügelasten, Mitteilungspflichten. Nicklisch und Weick (Fn. 7) Einleitung Rn. 4.

16 Helmut Schlotke (1987) »Mitwirkungspflichten bei Langzeitverträgen«, in: Fritz Nicklisch, Der komplexe Langzeitvertrag, Heidelberg: Müller, 377-390, 379; Volker Zerr (2002) »Gesamtschuldverhältnisse im Bauwesen «, 3 NZBau, 241-245, 241.

17 Nicklisch und Weick (Fn. 7) Einleitung Rn. 4.

18 Schlotke (Fn. 3) 381. 
handwerkliche Herstellung und Verarbeitung von Sachen ${ }^{19}$ und gilt für die Herstellung eines Maßanzugs wie einer Industrieanlage gleichermaßen ${ }^{20}$. Die Leistung des komplexen Langzeitvertrags ist es etwa, zu erklären, warum es für die Abnahme und Nachtragszahlungen anderer Vorschriften als die des BGB bedarf. Er sieht jedoch weder einen übergreifenden Zusammenhang des Vertragsgeflechts vor noch verlässt er die Bilateralität der Verträge in irgendeiner Weise.

\section{Strukturmerkmale des Franchising}

Das Franchising ist ein »vertikal-kooperativ organisiertes Absatzsystem rechtlich selbständiger Unternehmen auf der Basis eines Dauerschuldverhältnisses. « ${ }^{21}$ Das Ziel des Systems ist eine Effizienzsteigerung bei der Tätigkeit am Markt durch die kostensenkenden Effekte, die durch die Standardisierung von Warenproduktion, -vermarktung oder Dienstleistungserbringung erzielt werden. ${ }^{22}$ Der Franchisegeber schließt mit den Franchisenehmern gleichförmige Verträge ab. Diese verpflichten die Franchisenehmer auf Pflichtenstandards die dazu dienen, dass das System einheitlich am Markt auftritt. Zur Sicherstellung der Einhaltung der Systemstandards enthalten die Verträge ein Weisungs- und Kontrollsystem. Die Weiterentwicklung des Organisations- und Marketingsystems obliegt dem Franchisegeber. ${ }^{23}$ Der Franchisegeber schließt also strukturell gleiche bilaterale Verträge mit den Franchisenehmern ab. Er ist der einzige, der mit allen kontrahiert. Typischerweise treten keine wechselnden Abhängigkeitsverhältnisse zwischen globalen, regionalen und lokalen Einheiten oder Leistungsbeziehungen zwischen den Systembeteiligten untereinander auf. ${ }^{24}$ Das sich ergebende Netzwerk stellt sich als Stern dar. ${ }^{25}$ Es handelt sich um eine streng hierarchische Struktur, die zentrale Steuerungskompetenz ist dem Franchisegeber zugewiesen. Er ist die Zentrale, von der aus die Entscheidungsströme linear nach unten verlaufen.

Der Good Will des Netzes ist von der individuellen Leistungserbringung der einzelnen abhängig, da das System anfällig ist gegen Schäden, die durch Standardunterschreitung einzelner verursacht werden. ${ }^{26}$ Die synergetischen Wechselwirkungen können sich positiv oder negativ auf die Umsätze jedes einzelnen Franchisenehmers auswirken. ${ }^{27}$

19 Motive zu dem Entwurfe eines bürgerlichen Gesetzbuches für das Deutsche Reich II (1888) Berlin: Guttentag, 470; Hans-Leo Weyers (1981)»Werkvertrag« in: Bundesminister der Justiz (Hg.) Gutachten und Vorschläge zur Überarbeitung des Schuldrechts Bd II, Köln: Bundesanzeiger, 1115-1207, 1122.

20 Daran hat sich auch seit der Schuldrechtsreform nichts geändert, obwohl die Aufnahme von Vorschriften für den Bauvertrag vorgeschlagen wurde.

21 So ein Teil der Definition des Deutschen Franchiseverbands e.V.

22 Rohe (Fn. 2) 413 f.

23 Walther Skaupy (1992) »Zu den Begriffen ,Franchise', ,Franchisevereinbarungen' und ,Franchising' ", 45 NJW, 1785-1790, 1786; OLG Schleswig v. 27.08.1986, NJW-RR 1987, 220.

24 Rohe (Fn. 2) 357; Schimansky (Fn. 2) 108; Ralf Struthof (1999) Führung und Organisation von Unternehmensnetzwerken, Göttingen: Vandenhoeck und Ruprecht, 103 ff. zu heterarchischen Netzwerken.

25 Rohe (Fn. 2) 356 f.; Schimansky (Fn. 2) 108.

26 Rohe (Fn. 2) 415.

27 Schimansky (Fn. 2) 108. 


\section{Vergleich Baukooperation - Franchising}

Gemeinsam ist den beiden Netzwerktypen, dass es ein übergreifendes gemeinsames Projekt gibt. In der Baukooperation ist es die Erstellung des Bauwerks, beim Franchising die gemeinsame Absatzförderung. In beiden Netzwerken wird der übergreifende Zusammenhang entweder vertraglich gar nicht erwähnt, oder jedenfalls vertraglich nicht mit einer Wirkung ausgestattet. Die beiden Netztypen unterscheiden sich durch mehrere Faktoren. Der Unterschied liegt nicht darin, dass es sich beim Franchising mehr um eine »strategische Allianz« handelt, die Baukooperation dagegen nur von Sachzwängen beherrscht wird. Bauherr wie Franchisegeber könnten sich anderer Organisationsformen bedienen. Der Auftraggeber könnte sich genauso mit den Bietern zu einem Konsortium zusammenschließen, dessen gemeinsamer Zweck die Erstellung des Gesamtbauwerks ist, wie es der Franchisegeber könnte. Auch bei der Baukooperation ist die Wahl der Organisation der Verträge eine strategische Entscheidung. ${ }^{28}$ Ein Unterschied ergibt sich aus dem Zeitfaktor. Die Baukooperation ist in der Regel nicht auf Dauer angelegt, sondern wird durch das durchzuführende Projekt bestimmt. Das Netzwerk endet mit der Beendigung des Projekts, dessen Durchführung u.U. mehrere Jahre in Anspruch nehmen kann. Im Franchisesystem ist kein Auflösungszeitpunkt festgelegt. Es ist sozusagen ergebnisoffen. Ein Unterschied liegt auch in der Arbeitsweise. Während das Bauwerk arbeitsteilig hergestellt wird, wird die Absatzförderung mit Hilfe paralleler Leistungen erreicht. Schließlich unterscheiden sich die Vertragsnetzwerke in der Hierarchie. Franchisesysteme sind in der Regel streng hierarchisch aufgebaut mit gleichförmigen Pflichtenstandards. Der Franchisegeber trägt die Verantwortung für das reibungslose Funktionieren des Netzwerks. Zwar ist auch bei der Baukooperation der Auftraggeber je nach Vertragsgestaltung für die Koordinierung der Schnittstellen zuständig, wie gesehen werden Entscheidungen aber zumeist gemeinsam getroffen. ${ }^{29}$

Der eine Netzwerktyp ist der eines in der Dauer begrenzten, nicht ergebnisoffenen, arbeitsteiligen und eher heterarchischen Netzwerks, der andere ist der eines in der Dauer und im Ergebnis offenen, durch parallele Leistungen gekennzeichneten und hierarchischen Netzwerks. Die Unterschiede machen sich in den Rechtsfolgen der multilateralen Sonderverbindung bemerkbar. ${ }^{30}$

\section{Netzwerke als semi-spontane Ordnung}

Schlotke $^{31}$ gehört zu denjenigen, die in einem komplexen Langzeitvertrag einen rechtlichen übergreifenden Zusammenhang zwischen den bilateralen Verträgen erkennen, ohne sich zudem zur Begründung der Rechtsgeschäftslehre zu bedienen. Erforderlich ist nach Schlotke eine Betrachtung, die klar erkennen lässt, »wie sich ihre gesellschaftsrechtlich erhebliche - vielleicht zunächst so gar nicht gewollte - Wirkung wechselseitig verstärkt « ${ }^{32}{ }^{2}$ Gesellschaftsähnliche Bindungen sind auch dann gegeben, wenn jemand bei seinem Eintritt in das Gesamtgefüge komplexer Langzeitver-

28 Siehe oben unter Abschnitt I.

29 Siehe oben unter Abschnitt I.

30 Siehe unten unter Abschnitt V 1.

31 Schlotke (Fn. 3) 268.

32 Schlotke (Fn. 3) 268. 
träge einmal »A « sagt und dementsprechend gezwungen ist, » $\mathrm{B} \ll \mathrm{zu}$ sagen $\ll .{ }^{33} »$ Es geht darum, rechtstatsächlich auf Entwicklungen hinzuweisen, die nicht einmal vornehmlich Folge bewusster Machtausübung einzelner Unternehmer sind, sondern eher eigengesetzlich, häufig als Nebenwirkung, aus weitgehend außerrechtlichen immanenten Sachzwängen heraus erfolgen, bei denen die Rechtswirklichkeit häufig der rechtlichen Durchdringung, davonläuft'. « 34

Schlotke verwendet den Begriff nicht, aber er beschreibt nichts anderes als den Sachverhalt einer spontanen Ordnungsbildung.

\section{Spontane Ordnung Hayeks}

Die Terminologie der spontanen Ordnung geht auf F. A. von Hayek zurück. ${ }^{35}$ Hayek definiert eine Ordnung als einen Sachverhalt,

»in dem eine Vielzahl von Elementen verschiedener Arten in solcher Beziehung zueinander stehen, dass wir aus unserer Bekanntschaft mit einem räumlichen oder zeitlichen Teil des Ganzen lernen können, richtige Erwartungen bezüglich des Restes zu bilden, oder doch zumindest Erwartungen, die sich sehr wahrscheinlich als richtig erweisen werden. $\ll^{36}$

Hayek unterscheidet zwischen gemachten und spontanen Ordnungen. Eine gemachte Ordnung (taxis) zeichnet sich dadurch aus, dass sie von jemandem entworfen wurde und dessen Zwecke verfolgt. Sie wird von Hayek auch als Organisation bezeichnet. Eine spontane Ordnung (kosmos) ist demgegenüber eine Ordnung, die entstanden ist, ohne dass sie irgendjemand bewusst entworfen hat. ${ }^{37}$ Die Ordnung ist zweckfrei, denn die Ziele denen sie dient, sind die partikulären Ziele aller ihrer Teilnehmer in aller ihrer Vielfältigkeit und Gegensätzlichkeit. ${ }^{38}$ Sie ist Ergebnis menschlichen Handelns, aber nicht menschlichen Entwurfs. ${ }^{39}$ Dahinter steht zum einen die Vorstellung, dass eine Ordnung nicht nur von Kräften außerhalb eines Systems, also

33 Schlotke (Fn. 3) 274.

34 Schlotke (Fn. 3) 268.

35 Er bezieht sich auf Michael Polanyi (1951) The logic of liberty, London: Routledge, 159; Friedrich von Hayek (1991) Die Verfassung der Freiheit, Tübingen: Mohr \& Siebeck, 193. Hayek sprach aber bereits 1937 in Economia vom Preissystem als einem spontanen Prozess, Jens Schäfer (1999) Evolutionärer Erklärungsansatz von v. Hayek über die Entstehung von Organisationen, Aachen: Shaker, 77.

36 Friedrich von Hayek (1980) Recht, Gesetzgebung und Freiheit, Bd. 1 Regeln und Ordnung, München: Verlag moderne Industrie, 57.

37 Als Beispiele nennt Hayek Sprache, Gesetz, Moral und den Markt: Friedrich von Hayek (1975) «Die Anmaßung von Wissen«, 26 ORDO, 12-21, $20 \mathrm{f}$.

38 Friedrich von Hayek (1969) Freiburger Studien, Mohr \& Siebeck: Tübingen, 255. Diese Vielzahl der Tatsachen und Absichten ist ein einzelner Planer vorauszusehen nicht imstande. Hayek (Fn. 36) 59 ff.; ders. (Fn. 35) 42, 193.

39 Hayek (Fn. 38) 97 bezieht sich auf Adam Ferguson (1767) der geschrieben hat: »nations stumble upon establishments, which are indeed the result of human action, but not the execution of any human design «, An Essay on the History of Civil Society, Edinburgh: Edinburgh University Press, Part III, Kapitel 2. Erstausgabe abrufbar unter http:// www.ecn.bris.ac.uk/het/ferguson/civil.htm. 
exogen, geschaffen werden kann, sondern auch von innen, endogen bzw. selbst-erzeugend oder spontan. ${ }^{40}$ Zum anderen steht dahinter die epistemologische Annahme der Begrenztheit menschlichen Wissens, die es notwenig macht, dass sich die Individuen abstrakten Regeln unterwerfen. Hayek geht davon aus, dass die Verfolgung unterschiedlichster dezentraler und unabhängiger Zwecke durch die Individuen eine Ordnung aufweisen kann, wenn die Individuen regelmäßig in ihren Reaktionen auf ihre unmittelbare Umwelt spontan bestimmten Regeln folgen. ${ }^{41}$ Diese Handelnsregeln sind evolutionär ${ }^{42}$ herausgebildete Sitten, Gebräuche und Gewohnheiten, aber auch Rechtsregeln. ${ }^{43}$ Individuen befolgen Regeln, auch wenn sie keinen Zwangscharakter haben, weil sie diese zur Reduktion von Komplexität ihrer Umwelt benötigen. ${ }^{44} \mathrm{Da}$ der Mensch nicht fähig ist, »seine Handlungen im Wege einer vollständigen, expliziten Bewertung der Konsequenzen sämtlicher möglicher Alternativentscheidungen und bei vollständiger Kenntnis aller Umstände erfolgreich zu koordinieren $«,{ }^{45}$ bedarf er bei jeder Entscheidung einer Begrenzung seines Wahlbereichs mittels abstrakter Regeln. ${ }^{46}$ Es ist nach Hayek das einzige Werkzeug des Menschen, um seinem Handeln eine gewisse Kohärenz zu geben. ${ }^{47}$ Dabei setzen sich evolutorisch nur die Regeln gegenüber anderen Verhaltensmustern durch, die ein gesellschaftlich ausdifferenziertes Leben, die spontane Ordnung, ermöglichen und sichern. ${ }^{48}$ Die Ordnung muss wohltätig sein,

40 Die üblichen autoritären Assoziationen mit dem Begriff der Ordnung beruhen v.a. auf exogenen Verständnis von Ordnung, Hayek (Fn. 36) 57 f.

41 Die Regeln müssen nicht unbedingt in artikulierter Form vorhanden sein, Hayek (Fn. 36) 65.

42 Dies ist Hayeks grundsätzliche »Zwillingsidee von Evolution und spontaner Ordnung « (Fn. 38) 128, 156; ders. (Fn. 36) 40. Hayek verwendet die Entstehung von spontanen Ordnungen und spontaner Entstehung von Verhaltensregeln meist synonym: Die Regelentwicklung innerhalb spontaner Ordnungen vollzieht sich analog der Entwicklung der spontanen Ordnung selbst, als das Ergebnis eines evolutorischen Prozesses. Sie kann aber auch aufgrund konstruierter Regeln entstanden sein, ohne ihren spontanen Charakter zu verlieren, Viktor Vanberg (1981) Liberaler Evolutionismus oder vertragstheoretischer Konstitutionalismus? Zum Problem institutioneller Reformen bei F. A. von Hayek und J. M. Buchanan, Tübingen: Mohr \& Siebeck, 14, $86 \mathrm{f}$.

43 Zum Verhältnis dieser abstrakten Regeln: »Alle diese Regeln bestimmen zusammen mit den konkreten Tatsachen des Augenblicks die konkreten Entscheidungen des einzelnen und damit auch die Handelnsordnung des Ganzen. (...) Die Besonderheit, die die Rechtsregeln von anderen Verhaltensregeln unterscheidet, ist hauptsächlich, dass wir sie in einem gewissen Grade bewusst so gestalten können, dass sie in Verbindung mit anderen Regeln und in den zu erwartenden tatsächlichen Umständen zur Bildung einer Gesamtordnung führen, Friedrich von Hayek (1969) »Rechtsordnung und Handelnsordnung«, in: (Fn. 38) 177.

44 Friedrich von Hayek (1996) »Die Theorie komplexer Phänomene«, in: ders., Anmaßung von Wissen, Tübingen: Mohr \& Siebeck, 281-306. Vgl. auch den Ansatz von Niklas Luhmann (1989) Vertrauen: Ein Mechanismus der Reduktion sozialer Komplexität, 3. Aufl., Stuttgart: Ferdinand Enke.

45 Hayek (Fn. 38) 84.

46 Hayek (Fn. 38) 84.

47 Hayek (Fn. 38) 84.

48 Hayek (Fn. 36) 66 f. gibt das Beispiel, dass eine Regel, dass jeder Einzelne versuchen sollte, jeden, dem er begegnet zu töten, oder zu fliehen, wenn er einen anderen sieht, zu einer völligen Unmöglichkeit der Ordnung, in der die Tätigkeit des Einzelnen auf einer Zusammenarbeit mit anderen beruht, führte. 
da nur dann die Regeln allgemein und ohne Zwang befolgt werden. ${ }^{49}$ Hayek unterscheidet Regeln weiter in abstrakte und konkrete Regeln. Das Handeln der Akteure in spontanen Ordnungen beruht auf abstrakten Regeln (nomos), die für eine unbekannte Zahl zukünftiger Fälle und gleichermaßen für alle Personen gelten, auf welche die in der Regel beschriebenen objektiven Zustände zutreffen. In gemachten Ordnungen beruht nach Hayek das Handeln grundsätzlich auf konkreten Regeln, Befehlen, (thesis), die nur auf bestimmte Personen anwendbar sind oder den Zwecken des Organisators dienen. ${ }^{50}$

\section{Wettbewerb als Entdeckungsverfahren}

Spontane Ordnungen haben in Hayeks Theorie eine wichtige Funktion: Sie vermögen dezentrales, verstreutes und kontextuelles Einzelwissen aufzudecken. Wettbewerb als Entdeckungsverfahren ermöglicht den Mitgliedern der Gesellschaft ihre Ziele erfolgreicher zu verfolgen, als sie es nur mit ihrem individuellen Wissen könnten. ${ }^{51}$ Die Gesellschaft bedarf der Sammlung, Koordination und Verwertung von Wissen sowie der Generierung neuen Wissens. ${ }^{52}$ Hayek ist der Ansicht, dass die Verwertung des Wissens in der Gesellschaft am besten durch das Preissystem geleistet werden kann, das verborgene Informationen durch Veränderungen von Knappheitsverhältnissen, also steigende oder sinkende Preise, anzeigt und somit die durch Eigennutz gesteuerten Aktivitäten immer dorthin lenkt, wo steigende Preise eine Knappheit signalisieren. ${ }^{53}$ Der Gesetzgeber darf nach Hayek in die »natürliche Marktordnung « nicht intervenieren. Sie kann sonst ihre Koordinierungsleistung nicht erbringen, weil der Gesetzgeber nicht das vielfältige Einzelwissen umfasst und so das sachnahe, verstreute Einzelwissen verloren geht. ${ }^{54}$

Dies ist nach Hayek der Nachteil von Organisationen, die durch Befehle koordiniert werden. Befehle sind konkrete Regeln, die jemand mit nur begrenztem Wissen entworfen hat und dessen Zwecken dienen. Relevante Umstände und Informationen, die allein den Individuen bekannt sind, die aber für die Organisation von großem Interesse sein können, werden so nicht aufgedeckt. ${ }^{55}$ Von diesem Wissen können die Individuen in der Organisation nur Gebrauch machen, wenn sie in ihren Entscheidungen frei sind, was der Fall ist, wenn abstrakte Regeln ihr Handeln bestimmen. Auch Organisationen

49 Hayek (Fn. 36) 68. Das hat auch Cooter anhand eines Spiels nachgewiesen. Robert Cooter (1994) »Decentralized Law for a Complex Economy", 23 Southwestern University Law Review, 443-451, $445 \mathrm{f}$.

50 Hayek (Fn. 38) 110, 212.

51 Hayek (Fn. 35) 33.

52 Christopher Holl (2004) Wahrnehmung, menschliches Handeln und Institution, Tübingen: Mohr \& Siebeck, 125.

53 Zur Kritik an diesen Thesen, die etwa spekulative Prozesse (»Preise lügen«) außer Acht lassen, informativ, Karl-Heinz Brodbeck (2001) »Die fragwürdigen Grundlagen des Neoliberalismus«, 48 Zeitschrift für Politik, 49-71.

54 Hayek (Fn. 36) 75. Informativ hierzu Holl (Fn. 52) 124.

55 Hayek (Fn. 38) 41. 
müssen deshalb in gewissem Maße auf Regeln beruhen und können nicht allein durch spezielle Befehle geleitet werden. ${ }^{56}$

»In Organisationen komplexester Art wird kaum mehr als die Zuweisung bestimmter Funktionen an bestimmte Personen durch spezielle Entscheidungen bestimmt werden, während die Ausübung dieser Funktionen nur von Regeln gesteuert werden wird. $\ll^{57}$

\section{Semi-spontane Ordnung}

Die hier vorgestellten Netzwerke sind semi-spontane Ordnungen. Die Operationen der Beteiligten im Netzwerk beruhen zum einen auf den jeweiligen bilateralen Austauschverträgen, aber zugleich auch auf Regeln, die nicht vertraglich vereinbart sind und die kein einzelner festgelegt hat. Es sind Regeln, die von den Beteiligten im Rahmen ihrer jeweiligen individuellen Kenntnisse und Zwecke angewendet werden, die einem kollektiven Zweck entsprungen sind, der den Beteiligten nicht unbedingt in allen Einzelheiten bewusst ist. Die Leistungen werden so teils vertraglich, teils spontan erbracht. ${ }^{58}$ Der übergeordnete Zweck aller bilateralen Verträge, bzw. das gemeinsame Projekt, bringt Ordnung in das Netzwerk. ${ }^{59}$

»Im Gegensatz zu Hayeks spontaner Ordnung des Wettbewerbs als Entdeckungsverfahren sind hier gerade nicht Markt und Wettbewerb, sondern Vernetzung und Kooperation für die Ordnung der Spontaneität verantwortlich. « ${ }^{60}$

Da diese Ordnung nicht von der gänzlichen Abwesenheit von Zwecken gekennzeichnet ist, handelt es sich um eine semi-spontane Ordnung.

In Vertragsnetzwerken wie in Organisationen bestimmen sowohl gemachte als auch spontane Ordnung die Operationen der Beteiligten. Ein Vertragsnetzwerk ähnelt einerseits einer komplexen Organisation, in der der Auftraggeber oder der Franchisegeber wie der Organisator fungiert. Er ist es, der die Bildung des Netzwerks bilateraler Verträge initiiert. Er - im Gegensatz zu seinen Vertragspartnern - ist im Wesentlichen der Entwerfer des Franchisesystems bzw. Bauprojekts. Andererseits ist das Instrument zur Gestaltung einer Organisation im Privatrecht der Vertrag ${ }^{61}$ und dieses Mittel nutzen die Parteien nicht, um einen übergreifenden Zusammenhang zwischen den Einzelverträgen herzustellen. Die Vereinbarungen der Parteien in Netzwerken verbleiben streng im bilateralen Bereich. »Gemacht « ist in Netzwerken also nur eine Vielzahl bilateraler Organisationen, die die Schwelle zu einer übergreifenden Organisation nicht überschreiten. Die Beteiligten am Netzwerk bewegen sich aber auch nicht wie unabhän-

56 Hayek (Fn. 38) 212.

57 Hayek (Fn. 38) 42.

58 Teubner (Fn. 1) 127.

59 Die Begriffe des gemeinsamen Zwecks und des gemeinsamen Projekts werden hier synonym verstanden. Der Begriff des gemeinsamen Zwecks ist zwar vom Gesellschaftsrecht belegt, kann aber ein klares Unterscheidungsmerkmal zu anderen Kooperationsformen nicht leisten, siehe Fn. 66.

60 Teubner (Fn. 1) 133.

61 Er erzeugt eine gemachte Ordnung zwischen den beteiligten Parteien. So auch Schäfer (Fn. 35) 66. 
gige, in ihren Zielen und Plänen völlig unterschiedliche Akteure im spontanen Marktprozess, der die durch Eigennutz gesteuerten Aktivitäten immer dorthin lenkt, wo steigende Preise eine Knappheit signalisieren. Schlotke hat treffend beschrieben, wie sich gesellschaftsähnliche persönliche und vertrauensvolle Kooperationen jenseits bipolarer Bestimmungen entwickeln. Die Beteiligten an größeren Projekten missachten zunehmend formal erforderliche Leistungs-, Problem- und Mängelanzeigen und wirken immer intimer und formloser zusammen. Sie sehen ihren Auftrag zunehmend als Beitrag zur Problembewältigung und nicht als Teil vertragsgemäßer Leistungserfüllung und negieren so den Primat juristischer Aspekte der Bindung und Vertragsdurchführung. ${ }^{62}$ Zielbezogenes Zusammenwirken, Kommunikation ${ }^{63}$, wechselseitige Beobachtung, antizipatorische Anpassung, Vertrauen, Reziprozität, dauerhafter Beziehungszusammenhang, Selbstverpflichtung, Verhandlungen und Verlässlichkeit sind die Kennzeichen der spontanen Ordnung. ${ }^{64}$ Das sind nicht die Kennzeichen eines spontanen Wettbewerbsprozesses. Netzwerke stehen in einer Spannung, aber auch in einem Kontinuum, zwischen Markt und Organisation. Kennzeichen hierfür ist die Herausbildung eines übergeordneten Gesamtzwecks, eines Projekts, auf das das Handeln der Beteiligten und ihre Erwartungen über das Verhalten anderer ausgerichtet sind, ohne dass dies im einzelnen von jemandem bestimmt worden wäre. Bei der Ausführung ihrer vertraglichen Verpflichtung berücksichtigen die Beteiligten das Projekt, es überlagert jedoch nicht vollständig die individuellen Pläne und Zwecke der einzelnen. ${ }^{65}$ Es handelt sich um einen einheitlichen Zweck. ${ }^{66}$ Das Vertragsnetzwerk stellt einen Rahmen dar, der Freiraum lässt für Regeln statt Befehlen. Dies gilt vor allem in den »Maschen« des Netzwerks, in den Bereichen zwischen den vertraglich nicht verbundenen Beteiligten.

62 Achim-Rüdiger Börner (2000) »Netzwerke komplexer Langzeitverträge: Möglichkeiten des multilateralen Interessenausgleichs «, in: Fritz Nicklisch (Hg.) Netzwerke komplexer Langzeitverträge, München: Beck, 53-65, 56; vgl auch die Studie von Steward Macaulay (1963) »Non-Contractual Relations in Business: a Preliminary Study«, 28 American Sociological Review, 55-67 und abrufbar unter http://www.law.wisc.edu/facstaff/macaulay/ papers/non-contractual.pdf.

63 Schlotke (Fn. 16) 377, 379; Zerr (Fn. 16) 241.

64 Teubner (Fn. 1) 134.

65 Teubner (Fn. 1) 77 f. hat herausgearbeitet, dass es gerade der Witz von Netzwerken ist, dass simultan individuelle und gemeinschaftliche Zwecke verfolgt werden.

66 Die Abgrenzung von einem "gemeinsamen Zweck« des Gesellschaftsrechts und etwa einem »einheitlichen Zweck« in Vertragsnetzwerken (z.B. Andreas Saxinger (1993) Zulieferverträge im deutschen Recht. Baden-Baden: Nomos, 144; Schimansky (Fn. 2) 114 ff.) muss scheitern. Dies endet in gequälten Unterscheidungen, vgl. Nachweise bei Teubner (Fn. 1) 77 f. Teubner mutmaßt, dass hinter dem Unwohlsein der h.M. mit der Bejahung eines gemeinsamen Zwecks bei Netzwerken das Problem steht, dass simultan individuelle und gemeinschaftliche Zweckverfolgung vorliegen, wobei die individuelle Zweckverfolgung einen Stellenwert hat, der als gesellschaftswidriges Verhalten eingeordnet wird. 


\section{Gemischte Ordnungen bei Hayek}

Kann diese Konstruktion eines gemeinsamen Projekts, das eine übergreifende Ordnung auf ein Netz bilateraler Verträge stülpt, eine spontane Ordnung im Sinne Hayeks sein? Wie müssen die Regeln aussehen, damit eine spontane Ordnung entstehen kann? Wie muss die spontane Ordnung aussehen? Hayek bietet nicht nur die zwei Extreme spontane Ordnung und gemachte Ordnung - an, sondern erörtert auch Mischformen. Zusammenarbeit beruht nach Hayek stets auf bewusster Organisation und spontaner Ordnung. ${ }^{67}$ Netzwerke sind jedoch eine Kombination beider Ordnungen, zu der sich Hayek nicht geäußert hat. Es lassen sich Ausführungen Hayeks zu spontanen Ordnungen innerhalb der Binnenstruktur von Organisationen finden. Das sind etwa Kooperationsgruppen, die sich spontan innerhalb von Organisationen bilden. ${ }^{68}$ In Organisationen beruht nach Hayek das Handeln nicht nur auf Befehlen, sondern auch auf Regeln. Diese Regeln sollen sich jedoch von Regeln, auf denen eine spontane Ordnung beruht, unterscheiden:

»Regeln in einer Organisation setzen voraus, dass den einzelnen Personen durch Befehl bestimmte Aufgaben, Ziele oder Funktionen zugewiesen wurden, und die meisten Regeln einer Organisation gelten nur für Personen, die mit bestimmten Verantwortungen betraut wurden. Die Regeln einer Organisation werden deshalb der Absicht nach niemals universal oder zweck-unabhängig sein, sondern immer jene Befehle ergänzen, durch welche die Rollen verteilt bzw. die Aufgaben oder Ziele vorgeschrieben werden. Sie dienen nicht der spontanen Bildung einer abstrakten Ordnung, in der jedes Individuum seinen Platz finden muss und ein geschütztes Gebiet abstecken kann. Der Zweck und der allgemeine Rahmen der Organisation muss durch den Organisator bestimmt werden. $\ll{ }^{69}$

Eine gemischte Ordnung ist nach Hayek nur dann spontan, wenn

»sie sich der spontanen Ordnungskräfte nicht nur (...) bedient, um die Lücken zu füllen, die in den Ziele und Struktur bestimmenden Befehlen offen gelassen werden, sondern sich für die Bildung der Gesamtordnung auf die spontanen Kräfte verlässt. « ${ }^{70}$

Zunächst ist zu betonen, dass die hier beschriebenen Netzwerke nicht die Schwelle zur Organisation überschreiten. Das gemeinsame Projekt bringt Ordnung in eine Vielzahl geordneter Zweierbeziehungen (bilaterale Verträge), ohne eine Organisation zu begründen. Die Regeln, die ordnend den übergreifenden Zusammenhang in Bezug auf

67 Hayek (Fn. 36) 69.

68 Hayek (Fn. 35) 182. »Ob Hayek selbst das Potential für gemeinschaftliche Bindungen im Nexus der Reziprozität, d.h. in einem Netzwerk von Austauschbeziehungen, das alle wechselseitigen Übereinkünfte einschließlich der politischen einschließt, tatsächlich voll erkannt hat «? fragt James Buchanan (1999) »Moral und Gemeinschaft in der offenen Ordnung des Marktes«, in: Viktor Vanberg (Hg.) Freiheit, Wettbewerb und Wirtschaftsordnung - Hommage zum 100. Geburtstag von Friedrich A. von Hayek, Freiburg: Rudolf Haufe, 13-36, 24.

69 Hayek (Fn. 38) 213. Robert Gordon (1994) »Hayek and Cooter on Custom and Reason”, 23 Southwestern University Law Review, 453-460, 455 hat zu Bedenken gegeben, dass es das Schicksal jeder abstrakten Regel ist, durch richterliche Anwendung denkbar konkret zu werden, weil sie dann immer in einem Kontext stehen.

70 Hayek (Fn. 38) 43. 
das gemeinsame Projekt herstellen, sind mit Regeln in einer Organisation vergleichbar. Sie sind an einem Zweck, dem Projekt, orientiert. Sie sind nicht gänzlich zweckfrei, aber im Unterschied zu Organisationen, in denen gerade der Zweck geplant ist und durch Befehle durchgesetzt werden soll, entsteht der übergreifende Zweck gerade erst durch die Spontaneität und bringt die Ordnung in das Netzwerk. Es handelt sich dabei nicht um die fremden Zwecke des Auftraggebers oder des Franchisegebers. Der Zweck ist allenfalls zurückzuführen auf die bilateralen Verträge, und dort sind die Parteien gleichberechtigt. »Es wäre lächerlich zu sagen, dass ich mit der Erfüllung eines Kontraktes dem Willen eines anderen gehorche $\ll .{ }^{71}$ Das Projekt ist eher ein Zweck im Sinne der Funktion des Netzwerks. Hayek bleibt im Verhältnis spontaner Ordnung und Zweck dunkel. Es heißt:

»Spontane Ordnungen brauchen nicht zu sein, was wir abstrakt genannt haben. (...) Am wichtigsten ist jedoch die Beziehung einer spontanen Ordnung zum Begriff des Zwecks. Da eine solche Ordnung durch Einwirkung von außen geschaffen worden ist, kann die Ordnung als solche auch keinen Zweck haben, obwohl ihre Existenz für die Individuen, die sich in einer solchen Ordnung bewegen, von großem Nutzen sein kann. Aber in einem anderen Sinne kann man sagen, dass eine solche Ordnung auf zweckmäßigem Handeln ihrer Elemente beruht, wobei natürlich ,Zweck' nicht mehr bedeutet, als dass ihre Handlungen darauf deuten, diesen die Erhaltung oder Wiederherstellung jener Ordnung zu sichern. $\ll^{72}$

In diesem Zusammenhang ersetzt Hayek den Begriff »Zweck« durch den Begriff $»$ Funktion $\ll{ }^{73}$ Freilich hat er dabei wieder abstrakte Ordnungen wie den Markt vor Augen, nicht unsere Netzwerke. Hayeks Theorie ist vor dem Hintergrund seines Themas zu sehen: Er widmet sich dem Kampf gegen Planwirtschaft und Marxismus und dem Schutz der »natürlichen Marktordnung «. Es geht um Ressourcenallokation, Anreiz- Steuerungs- und Verteilungsprobleme und gesellschaftliche Wohlfahrt. Das Thema hier sind spontane Regeln und verstreute Informationen, die für das Netzwerk relevant sind! Daher gilt das Augenmerk für die hier untersuchten Vertragsnetzwerke nicht abstrakten Märkten, sondern abgrenzbaren Netzwerken, die nicht Organisationen sind.

Hayeks Haltung gegenüber Zusammenschlüssen und Organisationen ist entsprechend ambivalent. ${ }^{74}$ Sie schließen Wettbewerb aus, indem sie ihre Binnenbeziehungen regeln und durch Kartelle die Funktionsfähigkeit des Wettbewerbs bedrohen. ${ }^{75}$ Für ihn haben sie außerdem den Beigeschmack der Verfolgung partikulärer Zwecke einzelner. So werden Organisationen und auch örtliche Gebräuche in seinen Schriften mal als gut, mal als reaktionär behandelt. Kleinere Einheiten, wie Märkte, die in Zweierbeziehungen, lokale Gebräuche etc. eingebettet sind, lässt Hayek außer Acht. ${ }^{76}$ Sie finden

71 Hayek (Fn. 35) 184.

72 Hayek (Fn. 36) 61.

73 Hayek (Fn. 36) 62.

74 Gordon (Fn. 69) 457.

75 Hayek (Fn. 35) 48.

76 Das hat Gordon (Fn. 69) herausgearbeitet. Er nennt das Beispiel der mittelalterlichen Gilden, $458 \mathrm{f}$. 
nur Beachtung, wenn sie durch Absorption den Weg von lokalen Regeln in das Recht etwa durch das common law gemeistert, sich evolutorisch durchgesetzt haben. ${ }^{77}$

Die Bedeutung von spontanen Ordnungen in kleinen Gemeinschaften wird dagegen von Gordon und Cooter herausgestellt. Sie respektieren die örtliche Begrenztheit und Partikularität von Gebräuchen. ${ }^{78}$ Cooter zieht daraus die Konsequenz, dass das gesetzte Recht nicht innerhalb dieser Beziehungen zur Anwendung gelangen soll, sondern der Anwendung bei Streitigkeiten mit Fremden, die nicht an der Gemeinschaft beteiligt sind, vorbehalten ist. ${ }^{79}$ Cooter liefert allerdings keine Anleitung, wie die relevante Gemeinschaft und die Mitgliedschaft hieran zu ermitteln ist. ${ }^{80}$ Diese Auffassungen halten jedenfalls die gänzliche Abwesenheit von Partikulärinteressen und Zwecken nicht als Merkmal von spontanen Ordnungen aufrecht. ${ }^{81}$ Gegenüber diesen spontanen Ordnungen gehen Netzwerke aber noch einen Schritt weiter, da sie nicht die möglichen beteiligten Personen nach ihrem Berufsstand erfassen, wie in Gilden, sondern nach ihrer Einbettung in ein Projekt. Das gemeinsame Projekt begrenzt die relevante »Gemeinschaft $\ll$.

\section{Kooperation in Netzwerken als Entdeckungsverfahren}

In Netzwerken dient nicht allein der Wettbewerb zur Aufdeckung von Wissen, das für die Beteiligten innerhalb des Netzwerks von Bedeutung ist, sondern auch Kooperation. Was Hayek nicht beachtet, ist, dass innerhalb von Kooperationsgemeinschaften modifizierte Bedingungen für die Aufdeckung von Wissen gelten müssen. ${ }^{82}$ In Netzwerken steht der Auftraggeber oder der Franchisegeber vor dem gleichen Problem, wie der Entwerfer einer Organisation. Er ist für die Effizienz des Netzwerks darauf angewiesen, dass die Beteiligten am Netzwerk Wissen nutzen, dass er selbst nicht besitzt. Auch die anderen selbständigen Unternehmen bedürfen dieses Wissens, das für ihre Arbeit von Bedeutung sein kann. Es geht um auf das Netzwerk begrenzte Wissen, das jeder einzelne Beteiligte am Netzwerk besitzt, aber nicht alle zusammen. Das Wis-

77 Hayek stellt grundsätzlich den Prozess der Regelevolution gegenüber gestalteten Regeln deutlich in den Vordergrund und geht grundsätzlich von einer Synonymität der Entstehung einer spontanen Ordnung und spontaner Entstehung von Regeln aus, so dass die Regeln, wie die spontane Ordnung selbst zweckunabhängig sein müssen. Schäfer (Fn. 35$) 67$.

78 Cooter (Fn. 49) 445f.

79 Damit überträgt er für die Bestimmung der anzuwendenden Normen die Unterscheidung des Privatrechts zwischen Vertrag und Delikt in solche Gemeinschaften, Gordon (Fn. 69) 457.

80 Das gibt Gordon (Fn. 69) zu bedenken, 457.

81 So auch Joseph Kaiser. Kaiser sieht im Cyberspace eine spontane Ordnung. Das diese Ordnung konstituierende Handeln der Individuen sieht Kaiser auch durch einen höheren Zweck, die Funktion des Systems, bestimmt an, was an der Spontaneität der Ordnung nichts ändere. Joseph Kaiser (1997) »Das Recht im Cyberspace - Eine spontane Ordnung noch ohne Hierarchie«, in: Herbert Haller (Hg.) Festschrift Günther Winkler, Wien: Springer, 397-409, 402.

82 Diesen Unterschied hat auch Mestmäcker für den Markt mit Unternehmen und den Markt in Unternehmen angedeutet, Ernst Mestmäcker (1999) in: Viktor Vanberg (Fn. 59) 227$268,236$. 
sen kann im Netzwerk nicht nur über Markt und Wettbewerb aufgedeckt werden, da beides nicht uneingeschränkt vorhanden ist. Zur Aufdeckung dieses Netzwerkwissens und zum Ausgleich von Informationsasymmetrien dient die spontane Kooperation im Netzwerk. Dazu ein Beispiel aus der Baukooperation: Jede Bauvertragspartei hat die Möglichkeit, Handlungen durchzuführen, die von der anderen Partei nicht beobachtbar sind (hidden action), oder auf Grundlage von Informationen zu handeln, die dem Vertragspartner nicht bekannt sind (hidden information) ${ }^{83}$. Für die besser informierte Partei kann daraus ein Anreiz zu moral hazard entstehen, d.h. sie wird bestimmte effiziente Handlungen unterlassen, wenn sie die Grenzkosten dieser Handlungen selbst zu tragen hat, während sich die Grenzerlöse auf beide Parteien aufteilen. Wenn der Auftraggeber nicht in der Lage ist, Verzögerungen des Bauvorhabens, die durch einen Auftragnehmer verursacht sind, von Verzögerungen zu unterscheiden, die nicht auf seinem Verhalten beruhen, dann hat der Auftragnehmer auch keinen Anreiz, alle ökonomisch gerechtfertigten Maßnahmen zur Beschleunigung des Bauvorhabens durchzuführen. ${ }^{84}$ Führt die Bauzeitverzögerung zu keinem Schaden des Auftraggebers, etwa weil die nachfolgenden Unternehmer den Zeitverlust wieder aufholen, wird der Aufraggeber auch keine Anstrengungen unternehmen, die Ursache der Verzögerung aufzudecken. Die Kooperation mit dem Nachunternehmer, der seine Leistung auf der Leistung des anderen aufbaut, wird dagegen die Verursachung der Verzögerung schnell klären, und zwar nicht nur weil dem Nachunternehmer hierdurch ein Schaden droht $^{85}$, sondern auch weil eine lokale Kooperations- und Kommunikationsbeziehung besteht. Genauso wird das Unterlassen anderer effizienter Handlungen Thema in der lokalen Kooperationsbeziehung sein. In Vertragsnetzwerken, in denen dogmatische »blinde Flecken « gegenüber den vertraglich unverbundenen Beteiligten bestehen, hilft Kooperation zur Aufdeckung von relevanten Informationen. Das Netzwerk gibt zudem Anreiz zu vertragsgerechtem Verhalten ausgerichtet auf das gemeinsame Projekt.

83 Thomas Eger (1997) »Möglichkeiten und Grenzen der sozialen Kooperation durch langfristige Verträge«, in Claus Ott (Hg.) Effziente Verhaltenssteureung und Kooperation im Zivilrecht, Tübingen: Mohr \& Siebeck, 184-208, 188.

84 Eger (Fn. 83) 188.

85 Bisher blieben die Bauunternehmer bei Abwicklung entlang der Vertragskette in vielen Haftungsfragen unbehelligt, weil dem Auftraggeber als Vertragspartner kein Schaden entstanden ist und die Rechtsprechung keinen Fall der Drittschadensliquidation oder der Erfüllungsgehilfeneigenschaft des Vorunternehmers angenommen hat. Nunmehr wird u.U. ein Entschädigungsanspruch gegen den Auftraggeber gem. § 642 BGB gewährt, BGH NZBau 2000, 187. Verhilft die allseitige Kooperation zu einer reibungslosen und beschleunigten Abwicklung der Austauschverträge, kommt dies nicht nur dem Besteller zugute, was von Torsten Schwarze (2003) Das Kooperationsprinzip des Bauvertragsrechts, Berlin: Tenea, 193 eingewendet wird. Besonders bei Pauschalangeboten hat der Auftragnehmer ein hohes Interesse an einer reibungslosen Abwicklung. Nicht nur die Kosten für Personal und Material sind Kalkulationsbasis für das vom Auftragnehmer vorgelegte Angebot für seine Werkleistungen. Dazu rechnet er auch die voraussichtliche Zeitdauer und die daraus resultierenden Vorhaltekosten etc. mit ein. 


\section{Multilaterale Sonderverbindung}

Die semi-spontane Ordnung ist - im Recht abgebildet - eine vertragslose multilaterale Sonderverbindung. ${ }^{86}$ Die Notwendigkeit der Stabilisierung der von ihr ausgelösten Erwartungen ist ihre Legitimation.

»Ordnung (...) bedeutet daher im Wesentlichen, dass das individuelle Handeln von richtiger Voraussicht geleitet ist und dass die Leute nicht nur wirksamen Gebrauch von ihrem Wissen machen, sondern auch mit großem Vertrauen voraussehen können, welche Zusammenarbeit sie von anderen erwarten können. $\ll^{87}$

Einige Überlegungen zur spontanen Ordnung kann man auf eine Zweierbeziehung übertragen, um zu erklären, warum die semi-spontane Ordnung der Netzwerke auch jenseits von vertraglicher Vereinbarung rechtliche Konsequenzen auslöst. Es ist nicht von der Hand zu weisen, dass jeder Austauschvertrag durch die Verfolgung eines Transaktionszwecks zu einer Ordnung der Zweierbeziehung führt. Durch den bilateralen Vertrag werden Handlungsalternativen begrenzt und Komplexität reduziert. Der Vertrag löst bei jedem Vertragspartner unabhängig vom konkret vereinbarten Vertragsinhalt weitere v.a. am Transaktionszweck ausgerichtete Erwartungen an den anderen Teil aus, die er an Außenstehende Dritte nicht hätte. Dies ist nicht zuletzt der Grund, warum der Vertrag eine Sonderverbindung ist. § 242 BGB, »Treu und Glauben«, ist die Generalklausel, die im Vertragsrecht die berechtigten Erwartungen an den anderen Vertragspartner mit rechtlicher Wirkung ausstattet. Wie wir gesehen haben, löst die semi-spontane Ordnung ähnliche Erwartungen an die jeweils anderen am Netzwerk Beteiligten aus, die, wenn sie am Projekt orientiert sind, richtig ${ }^{88}$ sind. Über $§ 242$ BGB kommen spontane Ordnungen wie z. B. Verkehrssitten in den bilateralen Vertrag. ${ }^{89}$ Das BGB verweist an drei Stellen auf Verkehrssitten: In § 151 BGB, $\S 157$ BGB und $\S 242$ BGB. Nach $\S 242$ BGB etwa, ist die Leistung so zu bewirken, »wie Treu und Glauben mit Rücksicht auf die Verkehrssitte es erfordern«. In der Verweisung des

86 Eine Sonderverbindung vertraglich unverbundener aber auf ein gemeinsames Ziel verbundener Akteure nehmen Teubner (Fn. 1) für das Franchising und Just-In-Time-Zuliefererbeziehungen, Horst Eidenmüller (1999) Unternehmenssanierung zwischen Markt und Gesetz, Köln: Otto Schmidt, 608 ff.: für die Beteiligten an einer außergerichtlichen Unternehmensreorganisation an. Diejenigen, die Netzwerktheorien kritisieren, tendieren dazu zu vergessen, dass die legitimatorischen Grundlagen anderer Sonderverbindungen, wie die der c.i.c. keineswegs geklärt sind. Auch wenn sie mittlerweile gesetzlich normiert ist, dient die Bestimmung ihrer Legitimationsgrundlage auch der Bestimmung ihrer Tatbestandsmerkmale, Grenzen und Rechtsfolgen. Dies kommt besonders zum Tragen, wenn es um die Übertragung der jeweils in einem Rechtsgebiet entwickelten Institute auf ein anderes Rechtsgebiet und der Weiterentwicklung der Tatbestandsmerkmale geht. So hat gerade die Abkehr der Rechtsprechung von der Wohl-und-Wehe-Formel beim Drittschutzvertrag wieder Diskussionen ausgelöst. Vgl. Nachweise bei Anna Ziegltrum (1992) Der Vertrag mit Schutzwirkung für Dritte, Vom Fürsorgeverhältnis zu »gegenläufigen Interessen«, Frankfurt: Lang, 117.

87 Hayek (Fn. 35)193.

88 Richtig i.S.v. Hayek (Fn. 36).

89 Peter Oestmann (2002) »Verkehrssitte, Privatautonomie und spontane Ordnung «, 85 KritV, 409-437. 
Rechts auf die Verkehrssitten liegt die Anerkennung des nicht staatlich gelenkten Rechtsverkehrs zwischen den Individuen. ${ }^{90}$ Der Weg über $§ 242$ BGB wäre für Netzwerke aber nicht der richtige, denn genauso wie der Drittschutzvertrag vermag diese Norm den übergreifenden Zusammenhang der semi-spontanen Ordnung nicht abzubilden. ${ }^{91}$

Nun kann man einwenden, solch ungeschriebenen Regeln und spontane Ordnungen ließen sich überall finden, nicht jede regelmäßige Regelbefolgung könne jedoch zu einer multilateralen Sonderverbindung führen. Verkehrssitten, die als spontane Ordnung angesehen werden können, begründen in der Tat keine Sonderverbindung etwa zwischen allen Kaufleuten. Um eine spontane Ordnung zu einer multilateralen Sonderverbindung zu machen, bedarf es eines ordnungsgebenden gemeinsamen Projekts bzw. übergreifenden Zwecks. Die Besonderheit hier besteht in der Kombination von vertraglicher Vereinbarung und spontaner übergreifender Ordnung durch das gemeinsame Projekt, das nicht nur die vielfachen Handlungsalternativen der Beteiligten sondern zugleich den Geltungsbereich der Ordnung begrenzt.

\section{Rechtsfolgen der Sonderverbindung für vertraglich unverbundene Beteiligte}

Die multilaterale Sonderverbindung generiert Pflichten, die auf das Projekt bezogen sind. Sie können als Netzpflichten bezeichnet werden. Es kann sich um Kooperations-, Treue- und Schutzpflichten handeln. Nur Netzpflichten haben übergreifenden Bezug und nur für sie haften die vertraglich unverbundenen Beteiligten direkt. Die (Austausch-)pflichten aus den bilateralen Verträgen sind hiervon zu unterscheiden. ${ }^{92}$ Hierzu einige Beispiele: In Bauverzögerungsfällen kommt es vor, dass der Vorunternehmer

90 Die Rechtsprechung definiert Verkehrssitten als »den Verkehr tatsächlich beherrschende Übung«. BGH 43 NJW 1990, 1723, 1724; RGZ 49, 157, 162. Oestmann hat nachgewiesen, dass das Bestehen einer behaupteten Verkehrssitte in Gerichtsverfahren nicht empirisch ermittelt wird, obwohl es sich nach der Rechtsprechung um Tatsachen handeln soll. In der Rechtsprechung herrscht eine Art Misstrauen gegenüber spontanen Ordnungen vor. Die Ausführungen in den Urteilen sind unsauber und oftmals lapidar und floskelhaft, so Oestmann (Fn. 89) 425. Weiter wurde das Verhältnis von Treu und Glauben zu Verkehrssitten niemals wirklich geklärt. Die verbreitete Ansicht lautet, dass die Verkehrssitten dem Wertungsmaßstab des § 242 BGB unterworfen sind. Oestmann hat untersucht, dass die in der gängigen Kommentarliteratur hierfür angeführten Urteile diese Ansicht jedoch nicht stützen, weil sie Treu und Glauben zum Großteil gar nicht erwähnen. Interessant ist auch, dass das Recht sich eines Verweises auf die »guten « Sitten enthält. Das müsste nach Oestmann eigentlich bedeuten, dass es sich um grundsätzlich alle tatsächlich üblichen Verhaltensweisen handelt, nicht nur die rechtsethisch guten.

91 Eidenmüller (Fn. 86) 601, der eine Sonderverbindung der vertraglich unverbundenen Beteiligten an einer außergerichtlichen Unternehmensreorganisation annimmt. Sinn der übergreifenden Verbindung soll es sein, gerade auch Akkordstörer und Trittbrettfahrer einzubeziehen und zur Kooperation zu verpflichten. Als Rechtsgrund der Sonderverbindung sieht Eidenmüller das hohe Maß an Interessenübereinstimmung aller bezüglich des Gelingens des Reorganisationsvorhabens. Vgl. auch Wernhard Möschel (1986) »Dogmatische Strukturen des bargeldlosen Zahlungsverkehrs«, 186 AcP, 211-236, 222; Rohe (Fn. 2) 114; Lange (Fn. 1) Rn. 397; Teubner (Fn. 1) 200.

92 So auch Teubner (Fn. 1) $156 \mathrm{ff} . ;$ Rohe (Fn. 2) (netzwirksame und bipolare Interessenwahrungspflichten) 437 f.; a.A. Kulms (Fn. 2) 188. 
etwa aufgrund von Lieferengpässen seine Leistung nur verzögert erbringen kann und der nachfolgende Unternehmer deshalb nur mit Verspätung mit seiner Leistung beginnen kann. Durch den Verzug mit seiner Leistung allein hat der Vorunternehmer aber noch keine auf das Netzwerk bezogene Pflicht verletzt. Die Behinderung des Nachunternehmers stellt sich als Reflex aus dem Vertragsverhältnis von Auftraggeber und Vorunternehmer dar. Es handelt sich um die Realisierung eines Risikos, das sich auch bei isolierter Ausführung seines Werks hätte einstellen können. ${ }^{93}$ Erst, wenn der Vorunternehmer etwa verspätet den Auftraggeber und die Nachunternehmer informiert, dann verletzt er seine Pflicht zur Kooperation, die sich aus dem Bezug auf das Gesamtprojekt ergibt. Bei mangelhafter Vorarbeit, auf der ein Nachunternehmer seine Leistung aufzubauen hat, kann man nach der Art des Mangels differenzieren. Bei reinen Ausführungsmängeln ohne Bezug zur Nachleistung kommt zur bloßen Pflichtverletzung gegenüber dem Auftraggeber noch kein Moment hinzu, dass diese explizit als Verletzung von vernetzten Pflichten erscheinen lässt. Dort verwirklicht sich ein Risiko, dass dem Unternehmer nach Maßgabe seiner vollen gewerblichen Verantwortung aus dem Austauschvertrag zugewiesen ist. Reine Ausführungsmängel liegen nur im Risikobereich der Vertragsparteien, bzw. durch die Garantiehaftung im Risikobereich des Unternehmers. Der Vernetzungsaspekt kommt erst dann zum Tragen, wenn bei der Vorarbeit der Bezug zum Gesamtbauwerk verkannt oder ignoriert wird. Dies ist zum Beispiel der Fall, wenn der Vorunternehmer den falschen Estrich für die vom Nachunternehmer bekanntlich zu verlegenden Fliesen verwendet. Die Vernetzung liegt in der gegenseitigen Verzahnung der Leistungen. So muss sich der Vorunternehmer informieren, welche Arbeiten der Nachunternehmer vornehmen wird und wie er auf seiner Leistung aufbauen wird. Hierüber muss ihn der Nachunternehmer aufklären. Diese Pflicht besteht aus der Sonderverbindung direkt gegenüber dem Vorunternehmer. Verletzt der Nachunternehmer diese, haftet er sowohl dem Vorunternehmer als auch dem Auftraggeber auf Ersatz der dadurch entstandenen Schäden. Dies gilt aber nur mit Einschränkungen, denn auch der Nachunternehmer hat seine netzbezogenen Kooperationspflichten einzuhalten. So darf er die Vorleistung auch nach Erfüllung seiner gegenüber dem Vorunternehmer bestehenden Informationspflichten nicht einfach unbesehen zur Grundlage seiner Leistung machen, sondern muss sie nach seiner Sachkunde kontrollieren. Mängel oder Bedenken muss der Nachunternehmer unverzüglich dem Auftraggeber und dem Vorunternehmer mitteilen. Diese Pflicht ist eine Netzpflicht, gegenüber deren zügiger Erfüllung der Nachunternehmer allen Beteiligten im Netz haftet.

Im Franchising kann über die Sonderverbindung das sog. Freeriding erfasst werden. Freerider unterschreiten die vorgegebenen Pflichtenstandards zur Senkung ihrer Kosten, um ihren individuellen Gewinn zu steigern, während die anderen Beteiligten den Qualitätsstandard halten. ${ }^{94}$ Sie können so dem gesamten System einen Imageschaden zufügen, der zu Umsatzausfällen führen kann. Die Standards sind Netzpflichten. In der Sonderverbindung haften Freerider den anderen Beteiligten für deren Schaden unter

93 Hans Ganten (1974) Pflichtverletzung und Schadensrisiko im privaten Baurecht, Bielefeld: Gieseking, 201.

94 Schimansky (Fn. 2) 118. 
Umständen direkt. Es ergibt sich beim hierarchischen Netzwerktyp jedoch ein Unterschied, wenn das Franchising, wie es in der Regel der Fall ist, streng hierarchisch organisiert ist. Dies rückt das Netzwerk in die Nähe der Organisation, und zwar der Gesellschaft, und schwächt die Spontaneität der Ordnung. Dem trägt die Sonderverbindung Rechnung, wenn man dem Franchisegeber als zentraler Steuerungskompetenz die Einhaltung der Standards und die Beseitigung von Pflichtverletzungen überlässt. Teubner hat eine Einschränkung von der Direkthaftung vorgeschlagen, die dem Gesellschaftsrecht entstammt, die actio pro socio. ${ }^{95}$ Sie ist eine Mitgliedschaftsklage, mit der das Einzelmitglied Sozialansprüche des Verbandes im eigenen Namen geltend machen darf, wenn die zuständigen geschäftsführenden Gesellschafter trotz Aufforderung untätig bleiben. ${ }^{96}$ Dies würde den Primat des Franchisegebers respektieren.

\section{Vergleich der Konstruktion mit einem Konsortium}

Mit den zahlreichen sich aus der Sonderverbindung ergebenden und gegenüber allen Beteiligten bestehenden Netzpflichten werden die Beteiligten rechtlich in erhöhtem Maße auf das Gesamtprojekt verpflichtet. Vermieden wird aber gleichzeitig eine Haftung für Schäden, auf dessen Entstehung die Beteiligten keinen Einfluss haben. Zugleich steigen durch Information und Kooperation die Einwirkungsmöglichkeiten auf die jeweils anderen Beteiligten und damit die Beherrschung des Risikos. Es resultiert zwar ein kompliziertes System von Verantwortungen und Pflichten, es ist aber nicht unrealistisch. Bau-Konsortien weisen ähnliche Strukturen auf. Das Konsortium übernimmt wie eine ARGE im Außenverhältnis die Durchführung des Bauauftrags als Ganzes. Im Innenverhältnis wird jedoch der Gesamtauftrag in einzelne Lose aufgeteilt und in Subunternehmerverträgen an die Konsorten vergeben, die sie als eigenständige unternehmerische Leistung in der Regel zu einem festgelegten Preis ausführen. Die Konsorten führen den Bauauftrag also nicht gemeinschaftlich aus, sondern unter Bündelung der verschiedenen Teilleistungen auf der Ebene des Konsortiums. ${ }^{97}$ Die Konsorten bringen weder Personal noch Sachmittel in das Konsortium ein. Das Konsortium bildet daher grundsätzlich kein Gesamthandsvermögen, abgesehen von der Forderung auf Vergütung gegen den Auftraggeber. ${ }^{98}$ Nach h.M. ${ }^{99}$ ist ein Konsortium als Gesellschaft bürgerlichen Rechts zu qualifizieren. Die Konsorten sind zugleich Gesellschafter und Auftragnehmer des Konsortiums, sie haben eine Doppelstellung. In ihrer Eigenschaft als Nachunternehmer tragen sie das volle Leistungs- und Vergütungsrisiko für die von ihnen eigenständig zu erbringende Werkleistung allein. Auf der Ebene des Konsortiums sind sie als Gesellschafter auf das Ziel der

95 Teubner (Fn. 1) $208 \mathrm{f}$.

96 Uwe Hüffer (2003) Gesellschaftsrecht, München: Beck, § 10, 90.

97 Dirk Weitze (2003) Die Arbeitsgemeinschaft in der Bauwirtschaft, Frankfurt a. M.: Lang, 89.

98 Klaus Lionnet (1984) »Liefer- und Leistungskonsortien - Rechtliche Zuordnung und Risiken«, in: Fritz Nicklisch (Hg.) Bau- und Anlagenverträge - Risiken, Haftung, Streitbeilegung, Heidelberg: Müller, 121-133, 125; Burkhard Lotz (1996) «Der Konsortialvertrag des Anlagenbaus im In- und Ausland «, 20 ZfBR, 233-240, 234. Die Forderung ist gem. § 740 Abs. 1 S. 1 BGB nicht Teil des Auseinandersetzungsvermögens nach Auflösung der Gesellschaft. Sie ist daher kein typisches Gesellschaftsvermögen. 
Erstellung des Gesamtloses des Konsortiums als übergreifenden Zweck hin verbunden. ${ }^{100}$ Aus dem Zusammenschluss folgen eine Reihe von Gesellschafterpflichten, die nichts mit den einzelnen »Austauschleistungen« zu tun haben, so wie Kooperationsund Treupflichten. ${ }^{101}$ Die Parallelen zur multilateralen Sonderverbindung sind unverkennbar.

99 Peter Ulmer (2004) »vor § 705«, in: Franz Säcker und Kurt Rebmann (Hg.) Münchener Kommentar zum BGB, 4. Aufl., München: Beck, Rn. 80 f.; Walther Hadding (1991) »vor § 705«, in: Hans-Theodor Soergel (Begr.) Bürgerliches Gesetzbuch mit Einführungsgesetzen und Nebengesetzen, 12. Aufl., Stuttgart: Kohlhammer, Rn. 17 f.; früher Fritz Nicklisch (1985) »Rechtsfragen des Subunternehmervertrags bei Bau- und Anlagenprojekten im In- und Auslandsgeschäft«, 38 NJW, 2361-2370, 2364. Nach anderer Ansicht handelt es sich um einen gemischten atypischen Gesellschaftsvertrag, der gesellschaftsrechtliche Elemente enthält, die stark von werkvertraglichen Elementen überlagert werden, jetzt GbR: Fritz Nicklisch (2001) »Einleitung § 4-13«, in: Nicklisch und Weick (Fn. 7) Rn. 84.

100 Auch wenn darin nicht wirklich der Gesellschaftszweck liegen dürfte, denn eigentlich ist das Ziel der Dach-ARGE nur der Erhalt des Auftrags und dem entsprechenden Wunsch des Auftraggebers nach nur einem Vertragspartner nachzukommen.

101 Es ist zu überlegen, ob Konsortien überhaupt Gesellschaften sind, v.a. wenn sich die Bauunternehmer gegenseitig gegen das Ausfallrisiko mit Bürgschaften absichern. Dann kann kaum noch von einer Erfolgsgemeinschaft gesprochen werden, Barner (Fn. 10) 42. Für das Emissionskonsortium ebenso Harm Westermann (1967) »Das Emissionskonsortium als Beispiel der gesellschaftsrechtlichen Typendehnung «, 22 AG, 285-292, 291. 